\title{
Fluctuación poblacional de moscas de las frutas (Díptera: Tephritidae) en Nicaragua, basado en el sistema de vigilancia fitosanitaria, 2017
}

\section{Population fluctuation of fruit flies (Diptera: Tephritidae) in Nicaragua, based on the phytosanitary surveillance system, 2017}

\author{
Josué Danilo Saldaña Reyes', Juan Carlos Morán Centeno², Gregorio Varela Ochoa ${ }^{2}$ \\ ${ }^{1}$ Maestría en Ciencia en Medidas Sanitarias y Fitosanitarias. Instituto de Protección y Sanidad Agropecuaria (IPSA), ORCID: https://orcid. \\ org/0000-0002-4809-4689, josue.saldana@ipsa.gob.ni \\ ${ }^{2}$ Maestro en Ciencia en Agroecología y Desarrollo Sostenible. Universidad Nacional Agraria. Facultad de Agronomía, ORCID: https://or- \\ cid.org/0000-0001-6135-7271, juan.moran@ci.una.edu.ni \\ ${ }^{2}$ Maestro en Ciencia Agrícolas y Recursos Naturales. Universidad Nacional Agraria. Facultad de Agronomía, ORCID: https://orcid. \\ org/0000-0001-5158-4999, gregorio.varela@ci.una.edu.ni
}

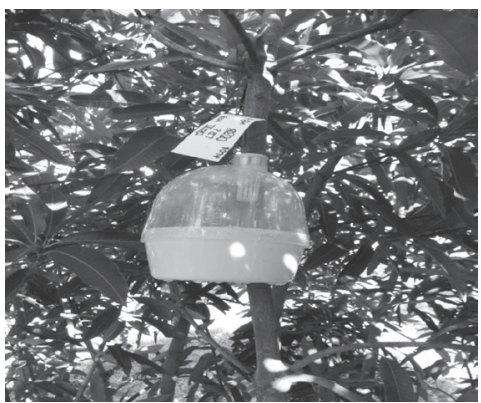

\section{RESUMEN}

El complejo de moscas de las frutas (Diptera: Tephritidae) representa uno de los principales problemas fitosanitarios a la producción frutícola de Nicaragua. Se efectuó el presente estudio con la finalidad de conocer la fluctuación del complejo de moscas de las frutas en Nicaragua, basado en el sistema de vigilancia fitosanitaria, en el periodo 2017, en un total de 17 rutas de trampeo a nivel nacional correspondiente a igual número de departamentos. Las trampas recibieron inspecciones y mantenimiento cada 15 días por especialistas del Instituto de Protección y Sanidad Agropecuaria (IPSA). Los datos se sometieron a análisis de estadística descriptivos. Las variables se analizaron, previa transformación $(\sqrt{x+0.5})$, mediante un análisis de la varianza (ANDEVA) de medidas repetidas. Para observar las diferencias entre las medidas pareadas se realizó un test de rangos múltiples de Tukey. Se evaluó número de adultos capturados, número de machos y hembras capturadas. El complejo de moscas de las frutas en Nicaragua está conformado por 10 especies, principalmente de los géneros Anastrepha (Nueve especies) y Ceratitis, las cuales están presente en todo el país, dándose las mayores capturas de hembras adultas. La fluctuación poblacional es variable en los departamentos del país, siendo los departamentos de Carazo, Jinotega y Nueva Segovia donde se reportaron las mayores cantidades de especímenes adultos, en los meses de abril, marzo y octubre. Las especies con mayor predominancia fue Anastrepha obliqua, Anastrepha serpentina, y Ceratitis capitata, en todas las rutas de muestreo en los diferentes meses del año y periodo evaluado.

Palabras clave: trampas, rutas de trampeo, Anastrepha, Ceratitis.

\section{ABSTRACT}

The fruit fly complex (Diptera: Tephritidae) represents one of the main phytosanitary problems for fruit production in Nicaragua. The present study was carried out with the determination of knowing the fluctuation of the fruit fly complex in Nicaragua, based on the phytosanitary surveillance system, in 2017, in a total of 17 trapping routes nationwide corresponding to the same number of departments. The traps received inspections and maintenance every 15 days by specialists from the Institute of Protection and Agricultural Health (IPSA). The data have been subjected to an analysis of descriptive statistics. The variables were analyzed, after transformation $(\sqrt{x+0.5})$, by means of an analysis of the variance (ANDEVA) of repeated measures. To observe the differences between the paired measurements, a Tukey multiple range test was performed. The number of adults captured, number of males and females captured was evaluated. The fruit fly complex in Nicaragua is made up of 10 species, mainly of the Anastrepha (Nine species) and Ceratitis genera, which are present throughout the country, giving the highest catches of adult females. The population fluctuation is variable in the departments of the country, being the departments of Carazo, Jinotega and Nueva Segovia where the largest numbers of adult specimens were reported, in the months of April, March and October. The most prevalent species were Anastrepha obliqua, Anastrepha serpentina, and Ceratitis capitata, in all sampling routes in the different months of the year and period evaluated.

Keywords: Traps, trapping routes, Anastrepha, Ceratitis.
Recibido: 8 de junio del 2019

Aceptado: 26 de septiembre del 2019

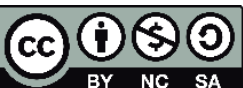
pueden obtener en el correo edgardo.jimenez@ci.una.edu.ni

Los artículos de la revista La Calera de la Universidad Nacional Agraria, Nicaragua, se comparten bajo términos de la licencia Creative Commons: Reconocimiento, No Comercial, Compartir Igual. Las autorizaciones adicionales a las aquí delimitadas se 


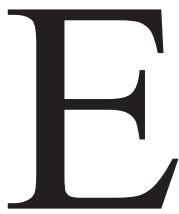

1 proceso de vigilancia fitosanitaria recoge y recopila información sobre la presencia o ausencia de una plaga (FAO, 2005). En Nicaragua la vigilancia se aplica sobre las plagas de mayor importancia en los cultivos nacionales, incluidos los programas de manejos (IPSA, 2000). En el contexto general, el complejo de la mosca de la fruta (Diptera: Tephritidae), según la OIEA, (2005) son la causa de pérdidas directas de muchas frutas y hortalizas. El complejo de mosca de la fruta representa un problema de carácter fitosanitario, debido a que éstas se encuentran distribuidas en áreas tropicales y subtropicales del mundo, estos dípteros son de importancia económica, utilizan las frutas como substrato para la oviposición y desarrollo de las larvas causando daños directos e indirectos en la fruticultura (Núñez, 2003).

Las moscas de las frutas pertenecen al Orden Díptera, Familia Tephritidae y comprenden aproximadamente 4000 especies descritas, de las cuales más de 400 se encuentran en el continente americano (Núñez, 2003). En Nicaragua el complejo de mosca de la fruta Anastrepha striata (Schiner) y especialmente, Ceratitis capitata (Wiedemann), son de importancia económica al afectar la producción de frutas destinadas a la exportación hacia Estados Unidos y Europa (IICA, 2005). Para el control se emplea el monitoreo y trampeo masivo. Las trampas utilizadas dependen del atrayente los que pueden contener feromonas sexuales. Los atrayentes para capturar hembras de mosca de la fruta se basan en alimentos o en olores del huésped.

Existe un alto potencial para el sector hortofrutícola, con excelente fertilidad de los suelos, estaciones climáticas bien definidas, precipitaciones favorables, acceso al riego, temperaturas adecuadas, ubicación geográfica, mano de obra abundante; admiten un desarrollo para los cultivos de hortalizas y frutas. Según INIDE (2012), existen 30083 productores dedicados a la actividad hortofrutícola, con un área de 30581 hectáreas. Estas áreas se establecen generalmente en pequeñas explotaciones.

La fluctuación poblacional se refiere a la cantidad de insectos que se encontraron en cada localidad, como respuesta a factores bióticos y abióticos, en un tiempo determinado (Begón et al., 1996). La disponibilidad de alimento es un factor de importancia en las fluctuaciones de las poblaciones, los factores climáticos y sus variaciones influyen en la cantidad de individuos en el tiempo, conocer la fluctuación es importante para determinar los periodos de mayor cantidad de individuos para tomar medidas de manejo y reducir las afectaciones a la producción (Ridgway, 2013). Conde-Blanco et al., (2018), afirma que el monitoreo de las poblaciones de moscas de las frutas permite tomar acciones de manejo que reduzcan los daños que estas ocasionan a la producción. Basado en la importancia de la producción hortofrutícola, se elaboró un estudio para conocer la fluctuación del complejo de moscas de las frutas (Diptera: Tephritidae) en Nicaragua, basado en el sistema de vigilancia fitosanitaria.

\section{MATERIALES Y MÉTODOS}

Ubicación del estudio. Nicaragua tiene un sistema de vigilancia fitosanitaria, el cual consiste en una red de trampas para plagas de interés económico. Parte del trabajo de investigación se efectuó en la identificación en laboratorio de especímenes proveniente de las 17 rutas de trampeo, que recibieron inspecciones y mantenimiento cada 15 días por especialistas del IPSA. De acuerdo al IPSA (2000) la disponibilidad de hospederos y la importancia y distribución de las trampas se realizó en la siguiente forma:

Puerto de entrada (Zona de alto riesgo $)=$ Cinco trampas por $\mathrm{km}^{2}$.

Zona rural (Zona de bajo riesgo $)=$ Una trampa por $\mathrm{km}^{2}$.

Vía de tráfico internacional = una trampa cada cinco $\mathrm{km}$.

Áreas comerciales $=$ Una trampa por hectárea

Descripción de la red de trampeo. Fueron establecidas 17 rutas de trampeo las que correspondieron a igual número de departamentos (Rosales, M, comunicación personal, 2 de diciembre de 2018), dentro de las cuales se usaron trampas de tipo McPhail cebadas con atrayente alimenticio (Torula/Bórax $\left.{ }^{\circledR}\right)$, para la captura de moscas del género Anastrepha spp; Ceratitis capitata (Wiedemann) y Tram-

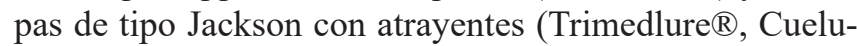
re $\AA$, Metileugeno $($ ), para capturas de machos los géneros C. capitata (Wiedemann). Se instalaron un total de 456 trampas, 110 trampas de tipo McPhail con atrayente Toru-

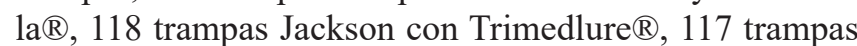
Jackson con Cuelure $\AA^{\circledR}$ y 111 trampas Jackson cebadas con Metileugeno® (Cuadro 1). 
Cuadro 1. Distribución de trampas y atrayentes en los departamentos de Nicaragua

\begin{tabular}{lccccc}
\hline & \multicolumn{5}{c}{ Tipo de trampa y atrayente } \\
\cline { 2 - 6 } Departamento & McP & TJ & & & \\
& TY & TML & TJ CU & TJ ME & Total \\
\hline Matagalpa & 6 & 7 & 5 & 6 & 24 \\
RACCN & 10 & 10 & 10 & 12 & 42 \\
RACCS & 21 & 20 & 20 & 17 & 78 \\
Rio San Juan & 9 & 15 & 15 & 12 & 51 \\
Rivas & 6 & 7 & 7 & 7 & 27 \\
Nueva Segovia & 6 & 6 & 6 & 6 & 24 \\
Masaya & 4 & 4 & 4 & 4 & 16 \\
Boaco & 4 & 4 & 4 & 4 & 16 \\
Carazo & 4 & 5 & 4 & 4 & 17 \\
Chinandega & 6 & 6 & 6 & 6 & 24 \\
Chontales & 4 & 4 & 4 & 4 & 16 \\
Estelí & 4 & 4 & 4 & 4 & 16 \\
Granada & 4 & 4 & 4 & 4 & 16 \\
Jinotega & 7 & 7 & 9 & 6 & 29 \\
León & 4 & 4 & 4 & 4 & 16 \\
Madriz & 5 & 5 & 5 & 5 & 20 \\
Managua & 6 & 6 & 6 & 6 & 24 \\
\hline Total & 110 & 118 & 117 & 111 & 456 \\
\hline
\end{tabular}

RACCN= Región Autónoma Costa Caribe Norte, RACCS $=$ Región Autónoma Costa Caribe Sur, McP TY= Mcphail cebada con torula, $\mathrm{TJ} \mathrm{TML}=$ Jackson cebadas con Trimedlure, $\mathrm{TJ}$ CU= Jackson cebadas con Cuelure y TJ ME= Jackson cebadas con Metil eugenol.

Identificación de especímenes. Los especímenes fueron recepcionados e identificados en el laboratorio de diagnóstico fitosanitario del programa área libre de moscas de la fruta del IPSA, usando claves taxonómicas (clave pictórica (López et al., 2010), las especies del genero Anastrepha Schiner. Las muestras fueron preservadas en alcohol al $70 \%$. Los resultados se organizaron en formato electrónico y se detalló la predominancia de cada una de las especies del complejo por departamento.

\section{Variables evaluadas}

Número de adultos capturados. Conteo directo en los diferentes tipos de trampas con cada atrayente evaluados.

Hembras capturadas. Fueron contabilizados y recepcionados cada 15 días en el laboratorio la identificación en los diferentes tipos de trampas, cada atrayente evaluados y cada especie.

Machos capturados. Contabilizados y recepcionados cada 15 días en laboratorio posterior a su identificación en los diferentes tipos de trampas, para cada atrayente evaluados y cada especie.
Fluctuación poblacional. Número de especies del complejo de moscas de las frutas en los departamentos bajo estudio, en el periodo evaluado.

Análisis de los datos. El manejo de los diferentes muestreos se agrupó en meses, relacionándolo con los departamentos bajo estudio. Los datos se sometieron a un análisis de estadísticas descriptiva. Los datos de variables se analizaron, previa transformación (Raíz cuadrada +0.5 ), mediante un análisis de varianza de medidas repetidas y observar las diferencias entre las medidas pareadas se realizó un test de rangos múltiples de Tukey $(\alpha=0.05)$.

\section{RESULTADOS Y DISCUSIÓN}

Incidencia del complejo de la mosca de la fruta. Se encontró mayor presencia de hembras y machos, sin importar el mes de muestreo $(\mathrm{Pr}=0.6600)$. Al evaluar los departamentos se determinó que hay diferencias estadísticas significativas en las capturas de especímenes $(\mathrm{Pr}=0.0001)$, la interacción de los meses con los departamentos fue similar en la captura de ambos sexos sin importar la especie de moscas de las frutas $(\operatorname{Pr}=0.2170)$. Estudio efectuado por Carrasco (2015) en el departamento de Carazo reportó que las variaciones en las capturas dependen del atrayente empleado y del estado de madurez del hospedante, este autor afirma que la mayoría de los atrayentes utilizados en la captura y monitoreo del complejo de mosca de la fruta están enfocado en la captura de hembras que son las que ocasionan el daño en la producción y son responsables de la siguiente generación de mosca. Muñoz (2003), menciona que, al adicionar atrayente para macho a las trampas, la capacidad de captura de hembras disminuye, ya que las hembras requieren proteínas específicas durante el periodo de ovoposición (Cuadro 2).

El complejo de mosca de la fruta está constituido por diversas especies de mosca. El comportamiento de las especies en los meses de muestreo indicó diferencias en Anastrepha obliqua (Macquart), Anastrepha striata (Schiner), Anastrepha serpentina (Wiedemann), y Anastrepha distincta (Greene), el comportamiento fue diferente en los departamentos del país para las especies A. oblicua. A. striata, A. serpentina, A. distincta, Anastrepha alveata (Stone) y $C$. capitata, el análisis de la interacción de los meses de muestreo y departamentos mostró efecto significativo para las especies de mosca $A$. striata, A. serpentina y $A$. distincta (Cuadro 2). Esto obedece al manejo que realizan los productores de frutas en las zonas productoras aledañas en donde se ubicaron las trampas en las rutas de trampeo, siendo el principal manejo el uso de insecticidas químicos (Delgado, 2016; Nolasco y Iannacone, 2008) 
manifiestan que la fluctuación poblacional de moscas de la fruta varía según la temporada, ubicación, disponibilidad de frutas y manejo del productor.

Cuadro 2. Análisis de varianza por mes, departamento e interacción en la fluctuación de especies de moscas de las frutas en Nicaragua

\begin{tabular}{lccccc}
\hline Especies & \multicolumn{5}{c}{ Variables } \\
\hline & Mes & $\begin{array}{c}\text { Departa- } \\
\text { mento }\end{array}$ & $\begin{array}{c}\text { Mes*depar- } \\
\text { tamento }\end{array}$ & $\mathrm{R}^{2}$ & $\mathrm{CV}$ \\
\hline A. obliqua & 0.0010 & 0.0010 & 0.5300 & 0.88 & 84.41 \\
A. ludens & 0.4700 & 0.1940 & 0.9980 & 0.70 & 16.64 \\
A. striata & 0.0001 & 0.0001 & 0.0001 & 0.98 & 28.81 \\
A. serpentina & 0.0001 & 0.0001 & 0.0001 & 0.98 & 18.33 \\
A. distincta & 0.0001 & 0.0001 & 0.0001 & 0.96 & 47.26 \\
A. bistrigata & 0.7653 & 0.3631 & 0.9940 & 0.71 & 73.75 \\
A. alveata & 0.8044 & 0.0003 & 0.9980 & 0.79 & 13.40 \\
A. ampliata & 0.3766 & 0.9902 & 0.9980 & 0.66 & 22.84 \\
C. capitata & 0.4469 & 0.0008 & 0.9990 & 0.77 & 18.70 \\
\hline S. Pr 0.05 & $\mathrm{~S}$ &
\end{tabular}

Si Pr $\leq 0.05$ es significativo $(\infty=0.05)$, de lo contrario es no significativo $\mathrm{R}^{2}=$ Coeficiente de determinación, $\mathrm{CV}=$ Coeficiente de variación.

\section{Fluctuación poblacional del complejo de moscas de las}

frutas. Las capturas se agruparon por departamentos, para determinar el comportamiento de las especies del complejo de moscas de las frutas. Se determinó que A. oblicua, A. serpentina, A. alveta y A. ampliata, mostraron las mayores cantidades de individuos en los diferentes departamentos. Aluja $(1993,1999)$ indica que el comportamiento está relacionado con la disponibilidad de alimento y las condiciones ambientales, principalmente precipitaciones al ejercer un control natural de las moscas, esta aseveración es confirmada por Carrasco (2015), quien demostró que en el periodo con mayor cantidad de precipitación la captura de adultos de moscas se reduce considerablemente (Cuadro 3).

Se observó que los departamentos de Madriz, León, Chinandega, Estelí, Chontales, y La Región de la Costa Caribe Sur (RACCS), se capturó mayor cantidad de individuos de las especies $A$. obliqua, en cambio $A$. striata, $A$. distincta y $A$. serpentina tuvieron un comportamiento similar en los diferentes departamentos. A. alveata mostró un comportamiento diferente con mayor número de especímenes en Masaya, Madriz, Rio San Juan, Nueva Segovia y La Región de la Costa Caribe Sur (Cuadro 3).

Ortiz Ramírez (2002), en estudio realizado en Carazo y Masaya encontraron que la predominancia de la especie $C$. capitata, está relacionada a la disponibilidad de alimento. Así mismo hace mención que las condiciones climáticas son ideales para el desarrollo y proliferación de esta especie.

Cuadro 3. Comparación de los valores promedios en la captura de especies de moscas de la fruta en los departamentos de Nicaragua

\begin{tabular}{|c|c|c|c|c|c|c|}
\hline \multirow{2}{*}{$\begin{array}{l}\text { Depar- } \\
\text { tamento }\end{array}$} & \multicolumn{6}{|c|}{ Especies de moscas de las frutas } \\
\hline & A. ol & iata & $\begin{array}{l}\text { A. ser } \\
\text { tin }\end{array}$ & & $\begin{array}{l}\text { A. dis- } \\
\text { tincta }\end{array}$ & A alvete \\
\hline Madriz & 8.29 & 2.82 & 0.73 & $\mathrm{~b}$ & $1.70 \mathrm{a}-\mathrm{c}$ & 4.08 \\
\hline Managua & 1.48 & 0.70 & 0.70 & $\mathrm{~b}$ & 0.70 & 0.70 \\
\hline eón & $4.17 \mathrm{ab}$ & $1.17 \mathrm{~d}-\mathrm{f}$ & 0.73 & $\mathrm{~b}$ & $0.75 \mathrm{bc}$ & 0.73 \\
\hline Chinandega & $4.04 \mathrm{ab}$ & $0.96 \mathrm{~d}-\mathrm{f}$ & 0.86 & $\mathrm{bc}$ & $0.74 \mathrm{bc}$ & 0.78 \\
\hline Boaco & $2.76 \mathrm{ab}$ & 0.70 & 0.70 & $\mathrm{~b}$ & 0.70 & 0.78 \\
\hline Jinotega & 1.28 & $1.05 \mathrm{~d}-\mathrm{f}$ & 0.74 & $\mathrm{~b}$ & $0.91 \mathrm{bc}$ & 0.74 \\
\hline Masaya & 1.04 & $1.14 \mathrm{~d}-\mathrm{f}$ & 0.70 & $\mathrm{~b}$ & $0.73 \mathrm{bc}$ & $1.02 \mathrm{ab}$ \\
\hline Carazo & 0.74 & $0.97 \mathrm{~d}-\mathrm{f}$ & 0.80 & $\mathrm{bc}$ & $0.74 \mathrm{bc}$ & 0.70 \\
\hline stel & $4.94 \mathrm{ab}$ & 5.59 & 0.70 & b & $1.33 \mathrm{~b}-\mathrm{d}$ & 0.87 \\
\hline Chontales & $5.16 \mathrm{ab}$ & 0.89 & 0.70 & b & $0.75 \mathrm{bc}$ & $1.76 \mathrm{ab}$ \\
\hline $\begin{array}{l}\text { Rio } \\
\text { San Juan }\end{array}$ & 2.22 & $2.01 \mathrm{c}-\mathrm{e}$ & 0.78 & $\mathrm{bc}$ & 0.97 & $1.17 \mathrm{ab}$ \\
\hline $\begin{array}{l}\text { Nueva } \\
\text { Segovia }\end{array}$ & 7.98 & 3.28 & 0.70 & b & 2.75 & $2.28 \mathrm{ab}$ \\
\hline Granada & 1.44 & $2.07 \mathrm{c}-\mathrm{e}$ & 1.04 & bc & 0.10 & 0.70 \\
\hline Matagalpa & 1.56 & $1.37 \mathrm{~d}-\mathrm{f}$ & 0.70 & b & 1.10 & 0.80 \\
\hline Rivas & 1.61 & 0.70 & 0.70 & b & 0.70 & 0.70 \\
\hline RACCN & 2.12 & $2.27 \mathrm{~cd}$ & 0.83 & $\mathrm{bc}$ & 0.78 & 0.70 \\
\hline RACCS & $4.37 \mathrm{ab}$ & 7.69 & 1.62 & $\mathrm{a}$ & $1.50 \mathrm{ab}$ & $1.47 \mathrm{ab}$ \\
\hline
\end{tabular}

Medias con letras iguales no difieren estadísticamente (Tukey $\alpha=0.05$ ).

Al analizar la fluctuación poblacional de las diferentes especies de moscas de las frutas en los diferentes meses del año, se encontró que en abril, marzo y octubre se capturaron la mayoría de las especies reportadas a nivel nacional, en cuanto a la cantidad de capturas promedio de adultos fue en los meses de noviembre, mayo y julio, hasta con 99.92 especímenes. En cuanto al sexo la mayor cantidad de capturas correspondieron a hembras (Cuadro 4). Carrasco (2015), menciona que el sexo femenino es el de mayor interés de vigilancia al ser responsable de dar origen a la próxima generación de mosca y las causantes del daño directo a la producción al perforar los frutos y depositar sus huevos, para que las larvas se alimenten dentro del fruto provocando la caída y pérdidas directa al productor. 
Cuadro 4. Intervalos de confianza en las capturas de especies del complejo de mosca de la fruta en los diferentes meses del año

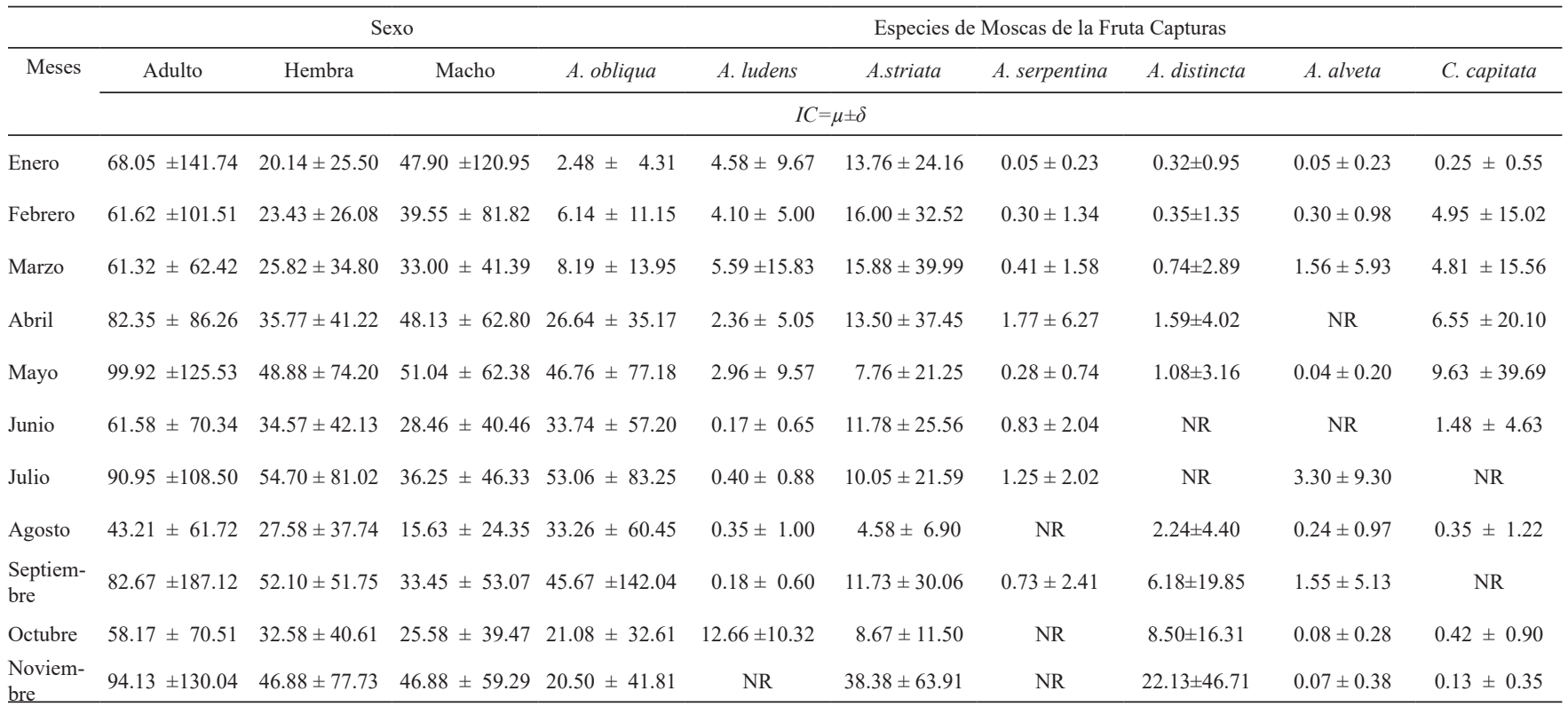

$\mathrm{IC}=\mu \pm \delta(\mathrm{IC}=$ Intervalo de confianza, $\mu=$ Promedio, $\delta=$ Desviación estándar $), \mathrm{NR}=$ No reporto captura.

En la Figura 1 se muestra el comportamiento de las diferentes especies, asociadas las capturas en los departamentos. C. capitata se encontró el mayor número de individuos en los departamentos de Carazo, Chinandega, Granada, Jinotega, León, Masaya y Estelí. A. obliqua, fue mayor la captura de adultos en Chinandega, Estelí, León, Madriz, Matagalpa y Rio San Juan. En lo concerniente a $A$. alveata, los departamentos de Nueva Segovia y Madriz con mayor frecuencia de individuos capturados, en Estelí, Madriz y La Región de la Costa Caribe Sur prevaleció la captura de $A$. striata, la especie $A$ distincta fue capturada en Madriz y Nueva Segovia. En Masaya A. ludens se capturó con mayor frecuencia, esto se relacionó

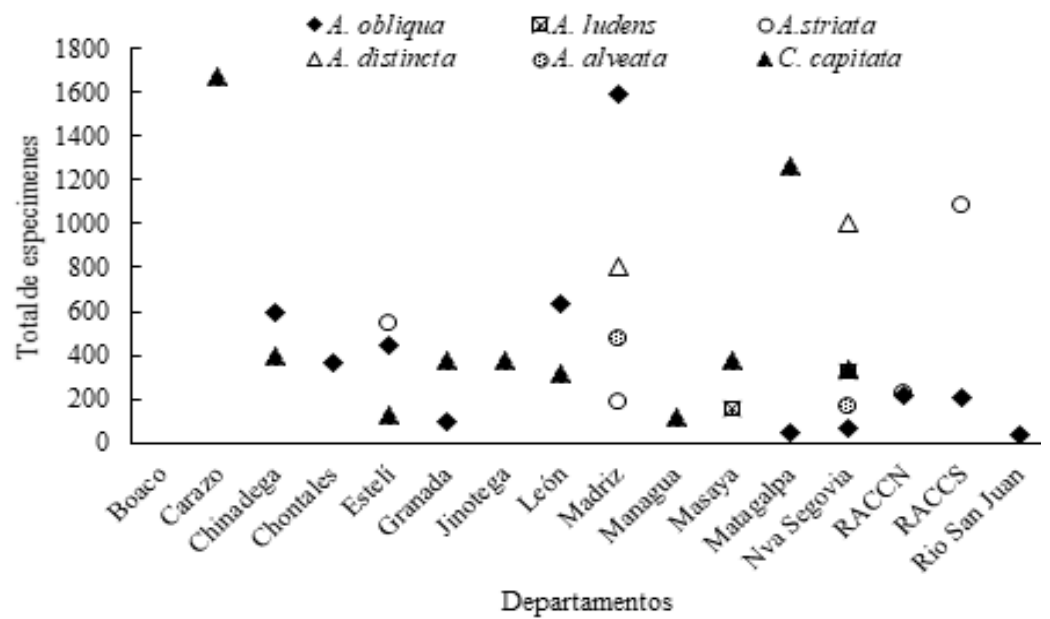

Figura 1. Fluctuación poblacional de adultos capturados de especies de moscas de las frutas, en los departamentos de Nicaragua.

\section{CONCLUSIÓN}

con la disponibilidad de alimento y las condiciones climáticas propia de cada departamento. Conde-Blanco et al., (2018), mencionan que el número total capturados es variable en las localidades y está estrechamente relacionado con la disponibilidad de alimento y las condiciones ambientales propia de cada región, Carrasco (2015) afirman que la relación de la captura de adultos con temperatura y precipitación es directa.

El complejo de la mosca de la fruta en Nicaragua está conformado por diez especies, principalmente de los géneros Anastrepha y Ceratitis, las cuales están presente en todo el país, dándose las mayores capturas de hembras adultas. La dinámica poblacional es variable en los departamentos del país, siendo el departamento de Carazo, seguido de Jinotega y Nueva Segovia en donde se reportaron las mayores cantidades de especímenes adultos. Las mayores capturas ocurrieron en los meses de abril, marzo y octubre. Las especies con mayor predominancia fue Anastrepha obliqua, Anastrepha serpentina, y Ceratitis capitata, en todas las rutas de muestreo en los diferentes meses del año. 


\section{REFERENCIAS BIBLIOGRÁFICAS}

Aluja Schuneman, M. (1993). Manejo integrado de la mosca de la fruta (No. SB608. F8 A47).

Aluja, M. (1999). Fruit fly (Diptera: Tephritidae) research in Latin America: myths, realities and dreams. Anais da Sociedade Entomológica do Brasil, 28(4), 565-594.

Begon, M., J.L. Harper \& C.R. Townsend. (1996). Ecology. Blackwel Science, Oxford. 1068p

Carrasco Rivera, L. C. (2015). Evaluación de trampas y atrayentes para el manejo de la mosca del mediterráneo (Ceratitis capitata Wied) con enfoque agroecológico, en el cultivo de mandarina (Citrus reticulata Blanco), la finca El Piñalito, San Marcos, Carazo (Dissertation, Universidad Nacional Agraria).

Conde-Blanco, E. A., Loza-Murguia, M. G., Asturizaga-Aruquipa, L. B., Ugarte-Anaya, D., \& Jiménez-Espinoza, R. (2018). Modelo de fluctuación poblacional de moscas de la fruta Ceratitis capitata (Wiedemann 1824) y Anastrepha spp (Díptera: Tephritidae) en dos rutas en el municipio de Caranavi, Bolivia. Journal of the Selva Andina Research Society, 9(1), 3-24.

Delgado Salmerón, A. L. (2016). Hymenoptera parasitoides asociados a Anastrpha spp (Díptera: Tephritidae) en frutales de San Francisco Libre y el Jicaral, Nicaragua (Dissertation, Universidad Nacional Agraria).

FAO. (Organización de las Naciones Unidas para la Alimentación y la Agricultura). (2005). Glosario de Términos Fitosanitarios. NIMF 5. p12.

IICA (Instituto Interamericano de Cooperación para la Agricultura. 2005. Productos agrícolas admisibles en los estados unidos. (D. Granda, Editor) Obtenido de Productos agrícolas admisibles en los estados unidos. Consultado en línea el 05 de marzo 2019. Disponible en http://repiica.iica.int/docs/B0415e/B0415e.pdf.

INIDE (Instituto Nacional de Información de Desarrollo). (2012). Informe final con los Resultados - a Nivel Nacional - del IV Censo Nacional Agropecuario (IV CENAGRO, 2011). 70 pág. Consultado en línea el 05 de marzo 2019 http://www.inide.gob.ni/Cenagro/ INFIVCENAGRO/IVCENAGROINFORME/assets/common/downloads/Informe\%20Final\%20IV\%20CENAGRO.pdf

IPSA. (Instituto de Protección y Sanidad Agropecuaria). (2000). Manual de Vigilancia Fitosanitaria. 36 P.

López, M. L., López, J. A., Hernández, E. A., Martínez, G. S., Gutiérrez, J. M., \& Hernández, R. A. (2010). Guía de campo para el reconocimiento de moscas de la fruta del género Anastrepha.

Muñoz, D. (2003). La mosca de la fruta (Ceratitis capitata) (Diptera: Tephritidae), en parcelas de cítricos; evolución estacional, distribución espacial y posibilidad de control, mediante trampeo masivo. Valencia, España. 196 Pag.

Nolasco, N., \& Iannacone, J. (2008). Fluctuación estacional de moscas de la fruta Anastrepha spp. y Ceratitis capitata (Wiedemann, 1824) (Diptera: Tephritidae) en trampas McPhail en Piura y en Ica, Perú. Acta zoológica mexicana, 24(3), 33-44.

OIEA (Organismo internacional de energía atómica). (2005). Guía para el trampeo en programas de control de la mosca de la fruta en áreas amplias. Viena, Austria. $48 \mathrm{P}$.

Ortiz Ramírez, C. A. (2002). Fluctuación poblacional de la mosca del mediterráneo (Ceratitis capitata (Wied.) en plantaciones de pitahaya (Hylocereus undatus (Britt y Rose) en los departamentos de Masaya y Carazo (abril 1999 a septiembre 2000) (Doctoral dissertation, Universidad Nacional Agraria, UNA).

Ridgway, R. (Ed.). (2013). Biological control by augmentation of natural enemies: insect and mite control with parasites and predators (Vol. 11). Springer Science \& Business Media. 